\title{
Uso de bifosfonatos en pacientes con osteopenia
}

\author{
Biphosphonates in patients with osteopenia
}

Sebastian Sguiglia

\begin{abstract}
Resumen
La osteopenia, una disminución de la densidad mineral ósea de menor severidad que la osteoporosis, definida por valores de T-score entre -1,0 y -2,5 en la densitometría ósea, podría asociarse con un mayor riesgo de fracturas. Motivado por el pedido de una paciente con osteopenia que solicita a su médico algún medicamento que le ayude a disminuir su riesgo de fracturas, el autor se pregunta si los bifosfonatos podrían ser beneficiosos para las pacientes con este factor de riesgo. Luego de realizar una búsqueda bibliográfica y seleccionar la evidencia más reciente y de mejor calidad, se concluye que estos fármacos podrían ser útiles para prevenir fracturas en mujeres mayores de 65 años con elevado riesgo de fractura, independientemente del resultado de la densitometría.
\end{abstract}

\begin{abstract}
Osteopenia, a minor decrease in bone mineral density, defined by T-score values between -1.0 and -2.5 in a bone densitometry, is associated with an increased risk of fractures. Moved by the request of a patient with osteopenia who asks her doctor for any medication that may help her reduce his risk of fractures, the author wonders if bisphosphonates could be beneficial for patients with this condition. After conducting a bibliographic search and selecting the most recent and best quality evidence, he concluded that these drugs could be useful to prevent fractures in women older than 65 years with a high risk of fracture, regardless of densitometry results.
\end{abstract}

Sguiglia S. Uso de bifosfonatos en pacientes con osteopenia. Evid Actual Pract Ambul. 2019;22(2):e001112.

\section{Escenario clínico}

Una paciente de 72 años de edad consulta a su médico de cabecera preocupada por el resultado de su densitometría ósea. Tiene un T-score de $-1,4$ en el cuello femoral. La paciente mide $161 \mathrm{~cm}$, pesa $60 \mathrm{~kg}$ y es tabaquista. No consume alcohol. Su madre se fracturó la cadera cuando tenía 80 años.

El riesgo de fractura osteoporótica a 10 años según la herramienta de evaluación de riesgo de fractura $\operatorname{FRAX}^{1}$ es de $12 \%$, y el riesgo de fractura de cadera es $6,6 \%$.

La paciente pregunta si hay algún medicamento que le ayude a disminuir su riesgo de fractura.

\section{Pregunta que generó el caso}

En mujeres posmenopáusicas con osteopenia y riesgo de fracturas elevado, ¿el uso de bifosfonatos es útil para prevenir la aparición de fracturas?

\section{Estrategia de búsqueda}

Se realizó una búsqueda en PubMed utilizando los términos Mesh: "Biphosphonates" y "Fractures, Bone", y el término de texto libre: "osteopenia". Se utilizaron los filtros para ensayos clínicos aleatorizados, revisiones sistemáticas y meta-análisis.

Se encontraron 18 referencias, las cuales no resultaban relevantes para la pregunta en cuestión.

Por otro lado, se consultaron las guías de práctica clínica del Colegio Americano de Médicos ${ }^{2}$ y de la Fundación Nacional para la Osteoporosis norteamericana ${ }^{3}$ y sus referencias. Se halló un análisis retrospectivo de cuatro ensayos clínicos que utilizaron risedronato ${ }^{4}$.

Además, se encontró un ensayo clínico aleatorizado adicional de publicación reciente ${ }^{5}$ que abordaba directamente la pregunta clínica.

\section{Algunas consideraciones sobre la osteoporosis y la osteopenia}

La osteoporosis es una condición clínica que se caracteriza por una disminución de la masa ósea y una alteración de su microarquitectura y que constituye un factor de riesgo para la aparición de fracturas ${ }^{2,6}$.

Se define por la presencia de fracturas por fragilidad o bien por un T-score de densidad ósea menor a -2,5. Se estima que aproximadamente 200 millones de personas presentan osteoporosis en el mundo. En Latinoamérica las tasas de fractura de cadera en hombres y mujeres mayores de 50 años es de 53 a 443 por 100.000 habitantes en mujeres, y de 27 a 135 por 100.000 habitantes en hombres, con una relación de 2 a 3 mujeres por hombre.

La osteopenia, por otro lado, se define por un T-score entre $-1,0$ y $-2,5$. Al igual que la osteoporosis, se encuentra asociada a un mayor riesgo de fracturas. Los pacientes con osteopenia tienen mayor probabilidad de progresar a osteoporosis. La prevalencia de osteopenia es mayor que la de osteoporosis, y se estima que un $50 \%$ de las fracturas ocurren en pacientes con densidad ósea en rango osteopénico.

El riesgo de fracturas de un paciente en particular se puede estimar utilizando puntajes como el FRAX, diseñado por la Organización Mundial de la Salud, que tiene en cuenta factores de riesgo clínicos para la aparición de fracturas como la edad, el género, el índice de masa corporal y el consumo de alcohol y tabaco, entre otros, y los resultados de la densitometría ósea (aunque este punto es optativo). Sin embargo, no hay evidencia de que la utilización del FRAX para basar decisiones terapéuticas mejore la prevención de fracturas ${ }^{2}$.

Existen muchos tratamientos disponibles para la prevención de fracturas. Los ejercicios de fortalecimiento muscular tienen un impacto favorable en la masa ósea, sin embargo no hay evidencia de que prevengan la aparición de fracturas. Los ejercicios destinados a mejorar el equilibrio y las medidas para reducir el riesgo de caídas son efectivas. Otras medidas recomendadas son la cesación tabáquica y evitar el consumo excesivo de alcohol.

La suplementación con calcio y vitamina $D$ es controvertida y no hay evidencia suficiente para sustentar su uso en las personas con osteoporosis; sin embargo, se recomienda la exposición solar y una ingesta adecuada de estos nutrientes.

a Servicio de Medicina Familiar y Comunitaria, Hospital Italiano de Buenos Aires. sebastian.sguiglia@hospitalitaliano.org.ar 
El tratamiento farmacológico está recomendado en términos generales en pacientes con osteoporosis y en quienes tuvieron una fractura por fragilidad previa. Las recomendaciones acerca del tratamiento farmacológico en pacientes con osteopenia se discuten más adelante (ver guías de práctica clínica)

Dentro de los tratamientos farmacológicos, los más utilizados son los bifosfonatos, drogas que inhiben el proceso de resorción ósea, aumentando indirectamente la densidad. Los más usados son alendronato, ibandronato o risedronato por vía oral en dosis diarias, semanales o mensuales. El zoledronato se utiliza por vía endovenosa, en forma anual.

La reducción del riesgo relativo de fracturas en pacientes que utilizan bifosfonatos, en comparación con placebo, es de 40 a $60 \%$ para fracturas vertebrales y 20 a $40 \%$ para fracturas no vertebrales.

Los efectos adversos más comunes de los bifosfonatos son los síntomas gastrointestinales leves. Deben utilizarse con precaución en personas con trastornos gastrointestinales previos y en aquellos con falla renal.

Existen efectos adversos raros, pero graves, como las fracturas atípicas del cuello femoral y la osteonecrosis de mandíbula. La incidencia de las fracturas atípicas es de 1,78 cada 100.000 personas cuando se toman por menos de 2 años y de 1 cada 1000 , cuando se toman por más de 8 años ${ }^{2,6}$. La relación riesgo/beneficio sería más favorable para el uso de bifosfonatos cuando se toman por 5 años o menos, ya que se estima que se produce una fractura atípica de fémur por cada cien fracturas evitadas.

La osteonecrosis de mandíbula es un evento raro (la incidencia es menor a 1 cada 10.000 pacientes) y es más frecuente con el uso prolongado y con los bifosfonatos endovenosos. Se recomienda una buena higiene dental y suspender el uso de estos fármacos antes de un procedimiento dental invasivo.

El uso de bifosfonatos también se asocia con efectos adversos musculares, oculares e hipocalcemia. La infusión de zoledronato puede ocasionar un síndrome pseudogripal. Por otro lado, no parece haber un aumento en la frecuencia de fibrilación auricular o de eventos cardiovasculares cuando se utilizan estas drogas.

\section{Guías de práctica clínica}

El Colegio Americano de Médicos recomienda fuertemente el tratamiento farmacológico con alendronato, risedronato, zoledronato o denosumab (en casos especiales) en pacientes con osteoporosis $^{2}$. La duración del tratamiento debería ser de cinco años, aunque la fuerza de esta recomendación es débil y se puede extender en pacientes con mayor riesgo de fracturas.

En cuanto a los pacientes con osteopenia, la recomendación de tratar es débil en mujeres mayores de 65 años, y la calidad de la evidencia es baja. El balance riesgo/beneficio puede ser mayor en pacientes con T-score más bajo o con riesgo clínico de fracturas más elevado.

Por su parte, la Fundación Nacional para la Osteoporosis norteamericana recomienda el tratamiento con bifosfonatos en pacientes con osteopenia y riesgo de fracturas osteoporóticas mayores de $20 \%$ o más, o de fractura de cadera de al menos $3 \%$, calculado mediante el FRAX. Estos puntos de corte se basan en un análisis de costo-efectividad realizado en los Estados Unidos.

Por último, la Asociación Argentina de Osteología y Metabolismo Mineral y la Sociedad Argentina de Osteoporosis proponen en su guía el mismo punto de corte para iniciar tratamiento farmacológico en la osteopenia ${ }^{7}$

\section{Resumen de la evidencia}

Se encontraron los siguientes trabajos, que evaluaron directamente el rol de los bifosfonatos en la prevención de fracturas en pacientes con osteopenia.
Otros ensayos no fueron tomados en cuenta porque se enfocaban en desenlaces intermedios, como por ej. el aumento de densidad ósea, o en poblaciones especiales como por ej. en trasplantados o pacientes con cáncer.

\section{1) Reid et al. Fracture prevention with zoledronate in older women} with osteopenia. NEJM 2018. 379:2407-2416 ${ }^{5}$

Métodos: Este ensayo clínico aleatorizado, doble ciego, de ácido zoledrónico contra placebo, se llevó a cabo en Australia entre 2011 y 2018. Se incluyeron mujeres posmenopáusicas de 65 años o más, con un T-score de -1 a $-2,5$ en la densitometría de cadera. Se excluyeron pacientes con insuficiencia renal, cáncer, enfermedades sistémicas importantes o uso de otros medicamentos que pudieran afectar el metabolismo óseo en el año previo al reclutamiento. No se excluyeron pacientes con osteoporosis de columna, salvo que el T-score fuera menor a -3 , o con osteoporosis en una de las caderas, siempre que la cadera contralateral estuviera en rango osteopénico.

El desenlace primario fue el tiempo transcurrido hasta la primera fractura por fragilidad. Se siguió a las pacientes mediante cuestionarios estructurados cada 3 meses y se les pidió que reportaran en forma inmediata cualquier fractura. También se midió la estatura de las pacientes y se obtuvieron radiografías de columna a los tres y a los seis años.

Los tratamientos consistieron en cuatro infusiones de ácido zoledrónico en dosis de $5 \mathrm{mg}$, separadas por intervalos de 18 meses, o placebo. Se administraron suplementos de vitamina $D$ a todas las pacientes y se aconsejó una ingesta adecuada de calcio. El análisis fue realizado por intención de tratar.

Resultados: Se incluyeron 2000 pacientes: 1000 en el grupo del zoledrónico y 1000 en el grupo placebo. Sus características basales, entre ellas la edad, el índice de masa corporal, la ingesta de calcio y el riesgo calculado de fracturas a 10 años, estuvieron repartidas en forma balanceada entre ambos grupos. Luego de seis años de seguimiento, se observaron fracturas en 190 mujeres del grupo control y en 122 del grupo activo (HR 0,63; IC95\% 0,5 a 0,79 ). El número necesario a tratar (NNT) para prevención de fracturas fue de 15. En un análisis de sensibilidad, se excluyeron a las pacientes con osteoporosis de columna o de una de las caderas $(\mathrm{N}=163)$ y en otro, a las que tenían un riesgo elevado de fracturas, sin que se modificase el resultado.

No se observaron diferencias en la mortalidad global y en la tasa de efectos adversos graves. Tampoco se reportaron casos de fracturas atípicas de fémur u osteonecrosis de mandíbula.

Conclusiones de los autores: El uso de ácido zoledrónico cada 18 meses fue útil para prevenir fracturas en mujeres con osteopenia, con una efectividad similar a la observada en pacientes con osteoporosis.

Fuentes de financiamiento: Público.

\section{2) Siris et al. Effects on risedronate on fracture risk in} postmenopausal women with osteopenia. Osteoporos Int 2008.19(5): $681-686^{4}$

Métodos: En un análisis retrospectivo de cuatro ensayos clínicos de risedronato contra placebo en pacientes con osteoporosis, se incluyeron a las pacientes que no tenían fracturas vertebrales previas y con una densitometría de cuello femoralcon T-score entre $-1,0$ y $-2,5$.

Todas las pacientes habían recibido suplementos de calcio y, si era necesario, de vitamina D. El desenlace fueron las fracturas confirmadas radiográficamente.

Resultados: Se incluyeron 620 pacientes aleatorizadas a risedronato $5 \mathrm{mg} / \mathrm{dí}$ ( $\mathrm{N}=311$ ) o placebo $(\mathrm{N}=309)$. Se observó un menor riesgo de fracturas en pacientes tratadas con risedronato 
(HR 0,27; IC95\% 0,09 a 0,83). Cuando se excluyeron las pacientes con osteoporosis de columna, el HR fue similar, sin embargo las diferencias no fueron estadísticamente significativas. No se observaron diferencias entre ambos grupos en cuanto a la incidencia de efectos adversos.

\section{Comentario}

Los bifosfonatos son una alternativa terapéutica útil para la prevención de fracturas en pacientes con osteoporosis. Los estudios resumidos sugieren que también podrían ser útiles en pacientes con osteopenia. Sin embargo, es importante tener en cuenta que el beneficio del tratamiento farmacológico dependerá del riesgo absoluto de sufrir fracturas, por lo que en las pacientes con bajo riesgo, el valor de la intervención es incierto.

El ensayo clínico de Reid et al. ${ }^{5}$, que muestra resultados muy prometedores, recibió fuertes críticas. Se señaló que casi un cuarto de las pacientes (24\%) tenían antecedentes de fracturas no vertebrales, por lo que en realidad tenían diagnóstico clínico de osteoporosis. Además, los autores no informaron determinadas características de las pacientes que podrían constituir factores de riesgo para sufrir caídas (como cataratas, glaucoma, disuria, enfermedades neurológicas, anemia, arritmias, frecuencia de caídas previas y medidas de la actividad de la vida diaria) y que podrían actuar como confundidores ${ }^{8}$. Por otro lado, también se destacó que la incidencia de fracturas observada en el estudio fue mayor a la esperada, lo que podría haber exagerado el efecto beneficioso del zoledronato ${ }^{9}$. Si bien los autores del estudio respondieron a estas y otras críticas ${ }^{10,11}$, sus resultados deberían interpretarse con cautela, teniendo en cuenta que el beneficio del tratamiento con bifosfonatos fue documentado en un grupo de pacientes con elevado riesgo absoluto de fracturas (alrededor del $20 \%$ en 6 años de seguimiento), que no es extrapolable al resto de las mujeres con alteraciones de la densidad mineral ósea den- tro de los valores que actulamente denominamos como osteopenia. En el caso de la paciente que originó la viñeta, cuyo riesgo de fractura estimado por FRAX es significativamente menor que el de las participantes incluidas en el estudio comentado, existe incertidumbre acerca de cuál podría ser el beneficio potencial si se le indican bifosfonatos.

Para tomar la decisión en conjunto con la paciente, es necesario proveer información completa, teniendo en cuenta otros aspectos del tratamiento farmacológico, como sus potenciales efectos adversos, incluyendo el riesgo de fracturas atípicas de fémur y de osteonecrosis de mandíbula, su costo económico, y las condiciones de administración de las drogas que determinan la practicidad del tratamiento. Existen herramientas de ayuda para las decisiones compartidas que deberían utilizarse para facilitar la comunicación de la información disponible y la toma de decisiones alineadas con los valores y preferencias de las pacientes ${ }^{11}$.

Por otro lado, es importante destacar la importancia de reforzar las estrategias para la prevención de caídas, ya que pueden evitarse muchas fracturas al reducir el riesgo de sufrir estos eventos (como el ejercicio físico regular, la reducción de medicación inapropiada, las pautas de seguridad en el hogar y la corrección de alteraciones visuales $u$ otros factores de riesgo específicos).

\section{Conclusiones}

En mujeres posmenopáusicas de 65 años o más con elevado riesgo de fracturas, el uso de bifosfonatos podría ser útil para prevenir fracturas independientemente del resultado de la densitometría (osteoporosis u osteopenia). Debería implementarse la toma de decisiones compartidas, teniendo en cuenta la mejor evidencia disponible y el riesgo de fractura de la paciente, y sin dejar de lado las estrategias de prevención de caídas.

\section{Referencias}

1. The University of Sheffield. FRAX ${ }^{\circledR}$ Herramienta de Evaluación de Riesgo de Fractura;. Available from: https://www.sheffield.ac.uk/FRAX/index. aspx?lang=sp [Last access: 2019-09-23].

2. Qaseem A, Forciea MA, McLean RM, Denberg TD, of the American College of PCGC. Treatment of Low Bone Density or Osteoporosis to Prevent Fractures in Men and Women: A Clinical Practice Guideline Update From the American College of Physicians. Ann Intern Med. 2017;166(11):818839. Available from: $10.7326 / \mathrm{M} 15-1361$.

3. Cosman F, de Beur SJ, LeBoff MS, Lewiecki EM, Tanner B, Randall S, et al. Clinician's Guide to Prevention and Treatment of Osteoporosis. Osteoporos Int. 2014;25(10):2359-81. Available from: 10.1007/s00198-014-2794-2.

4. Siris ES, Simon JA, Barton IP, McClung MR, Grauer A. Effects of risedronate on fracture risk in postmenopausal women with osteopenia. Osteoporos Int. 2008;19(5):681-6. Available from: 10.1007/s00198-007-0493-y.

5. Reid IR, Horne AM, Mihov B, Stewart A, Garratt E, Wong S, et al. Fracture prevention with zoledronate in older women with osteopenia. N Eng $\mathrm{J}$ Med. 2018;379(25):2407-2416. Available from: 10.1056/NEJMoa1808082.

6. Black DM, Rosen CJ. Clinical Practice. Postmenopausal Osteoporosis. N Engl J Med. 2016;374(3):254-62. Available from: 10.1056/ NEJMcp1513724.

7. Schurman L, Galich AM, Gonzalez C, Gonzalez D, Messina OD, Sedlinsky C, et al. [Argentine guidelines for the diagnosis, prevention and treatment of osteoporosis, 2015]. Medicina (B Aires). 2017;77(1):46-60.

8. Suzuki S, Hagino N, Nango E. Fracture Prevention with Zoledronate in Older Women with Osteopenia. N Eng J Med. 2019;380(13):1287. Available from: 10.1056/NEJMc1900923.

9. Tufan F. Fracture Prevention with Zoledronate in Older Women with Osteopenia. N Eng J Med. 2019;380(13):1287. Available from: 10.1056/ NEJMc1900923.

10. Reid IR, Horne AM, Gamble GD. Fracture Prevention with Zoledronate in Older Women with Osteopenia. Reply. N Eng J Med. 2019;380(13):1288. Available from: 10.1056/NEJMc1900923.

11. Mayo Clinic. Bone Health Choice;. Available from: https://osteoporosisdecisionaid.mayoclinic.org/index.php/site/index [Last access: 2019-09-23]. 